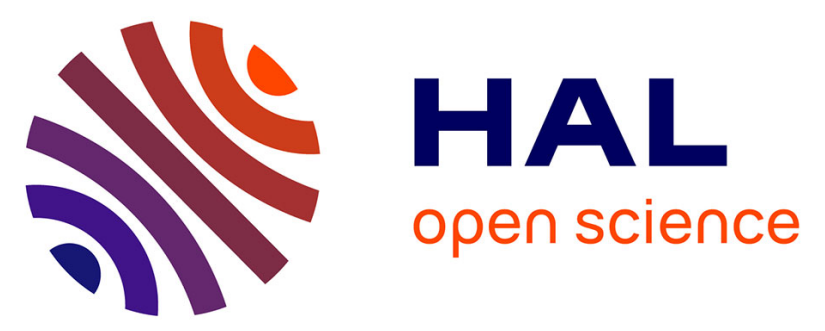

\title{
VIH-02 - Le dépistage de l'infection par le VIH : étude des pratiques des médecins généralistes en Martinique
}

B. Lafon-Desmurs, A. Criquet-Hayot, S. M'pay, A. Cabié

\section{To cite this version:}

B. Lafon-Desmurs, A. Criquet-Hayot, S. M'pay, A. Cabié. VIH-02 - Le dépistage de l'infection par le VIH: étude des pratiques des médecins généralistes en Martinique. 17es Journées Nationales d'Infectiologie, Jun 2016, Lille, France. Médecine et maladies infectieuses 46 (2016) 117-127, 46 (4). inserm-01410436

\section{HAL Id: inserm-01410436 https://www.hal.inserm.fr/inserm-01410436}

Submitted on 6 Dec 2016

HAL is a multi-disciplinary open access archive for the deposit and dissemination of scientific research documents, whether they are published or not. The documents may come from teaching and research institutions in France or abroad, or from public or private research centers.
L'archive ouverte pluridisciplinaire HAL, est destinée au dépôt et à la diffusion de documents scientifiques de niveau recherche, publiés ou non, émanant des établissements d'enseignement et de recherche français ou étrangers, des laboratoires publics ou privés. 


\section{$17^{\text {es }}$ Journées Nationales d'Infectiologie}

\section{Posters}

\section{VIH - Infections à VIH}

\section{VIH-01}

Typologie des nouveaux patients VIH dépistés annuellement et suivis dans un centre parisien entre 2010 et 2015 : évolution et perspectives

L. Fonquernie (1), N. Valin (1), M. Brucker (1), J. Bottero (1), L. Surgers (1), S. Fournier (1), P. Girard (1)

(1) CHU Saint-Antoine, Paris, France.

Introduction Description des nouveaux patients VIH dépistés annuellement et suivis dans un centre parisien sur la période 2010-2015 afin d'observer des tendances évolutives

Matériels et méthodes Les données épidémiologiques, clinico-biologiques et thérapeutiques sont extraites annuellement de la base informatique DIAMM ${ }^{\circledR}$ utilisée pour le suivi médical des patients

Résultats Entre 2010 et 2015, une moyenne de 161 patients VIH ont été annuellement dépistés et suivis dans le service, soit 4,5\% de la file active (967 au total). Plus de $50 \%$ des cas ont eu un dépistage systématique. En 6 ans, la prévalence du sous-type $\mathrm{B}$ est passée de $60 \%$ à $41 \%$. Le ratio $\mathrm{H} / \mathrm{F}$ était inchangé à 4 . L'âge moyen au diagnostic était stable à 37 ans dont près de $10 \%$ âgés de moins de 25 ans. Le mode de transmission était stable, homosexuel chez plus de $70 \%$ des hommes et hétérosexuel chez plus de $90 \%$ des femmes. L'origine géographique était comparable, européenne pour $65 \%$ des cas et subsaharienne pour $25 \%$. Près de $20 \%$ des cas était en primoinfection. Le sida inaugural (pneumocystose et tuberculose principalement) a chuté de $9 \%$ à $5 \%$ sur la période. Le taux moyen de CD4 initial était à $412 / \mathrm{mm}^{3}$. En 6 ans, la proportion de cas avec CD4 inférieur à $200 / \mathrm{mm}^{3}$ est restée voisine de $20 \%$. Le taux de patients restés naïfs à chaque fin d'année est passé de $50 \%$ à $23 \%$. A la mise sous traitement, le taux de moyen de CD4 est passé 379 à $420 / \mathrm{mm}^{3}$. En 6 ans, le choix de $1^{\text {re }}$ ligne d'antirétroviraux incluant comme $3^{\mathrm{e}}$ agent une IP boostée, un NNRTI ou une antiintégrase est passé de $70 \%$ à $17 \%, 16 \%$ à $11 \%$ et $14 \%$ à $71 \%$ respectivement. La prévalence de la coinfection B ou C est restée voisine de $5 \%$. Sur la période, le taux moyen de patients nouvellement diagnostiqués et non revus l'année suivante était de $15 \%$ dont près de $8 \%$ restaient perdus de vue

Conclusion Les patients nouvellement diagnostiqués reflètent la dynamique de l'épidémie VIH à court terme. En 6 ans, les HSH européens et les femmes africaines demeurent les 2 sous-groupes principaux. Près d'un quart des cas ont un taux de CD4 inférieur à $200 / \mathrm{mm}^{3}$ témoin d'un retard au diagnostic. La mise sous antirétroviraux est devenue plus précoce. Le choix initial d'une IP boostée a diminué alors que les anti-intégrases sont devenues prépondérantes

Aucun lien d'intérêt

\section{VIH-02}

Le dépistage de l'infection par le VIH : étude des pratiques des médecins généralistes en Martinique

B. Lafon-Desmurs (1), A. Criquet-Hayot (1), S. M'Pay (1), A. Cabié (1) (1) $\mathrm{CHU}$ de Martinique.

Introduction En France, la proportion de personnes infectées par le VIH et ignorant leur statut était de $25 \%$ en 2010 . On estime que ces personnes seraient à l'origine de $60 \%$ des nouvelles contaminations. Les offres et les moyens de dépistage sont régulièrement élargis et améliorés, sollicitant notamment les médecins généralistes. Cependant, les opportunités manquées de diagnostic sont nom- breuses : pour $82 \%$ des nouvelles découvertes de séropositivités VIH en 20092010. Une étude des pratiques de dépistage a eu lieu auprès des généralistes de métropole en 2009. Aucune étude de ce type n'a été réalisée en Martinique, département pourtant très touché par l'épidémie de VIH. Nous avons voulu réaliser un état des lieux des pratiques de dépistage de l'infection par le VIH par les généralistes en Martinique.

Matériels et méthodes Il s'agissait d'une étude descriptive réalisée par autoquestionnaire en ligne diffusé via une mailing-liste à 280 médecins généralistes sur les 285 recensés sur l'île. La durée de recueil était de 8 semaines. Les médecins et internes remplaçants étaient exclus de l'étude. Le questionnaire a été élaboré sur la base de l'étude « Baromètre Santé Médecins Généralistes » de 2009 qui étudiait les pratiques des généralistes sur plusieurs aspects, notamment le dépistage de l'infection par le VIH.

Résultats Nous avons obtenu 107 réponses, ce qui représentait 37,5\% des généralistes de la Martinique pratiquant une activité en cabinet. Ces médecins étaient d'importants prescripteurs avec une médiane de 10 tests VIH prescrits sur le dernier mois $(\mathrm{Q} 1=5 ; \mathrm{Q} 3=15)$. Ils avaient une opinion favorable du dépistage généralisé : entre $53,8 \%(\mathrm{n}=56)$ et $90,6 \%(\mathrm{n}=96)$. Près des deux tiers des praticiens $(63,5 \%, \mathrm{n}=66)$ étaient à l'initiative de la prescription du test. Certaines situations comme un contexte d'IST, de toxicomanie ou de prostitution engendraient une prescription théorique large : respectivement $72 \%(\mathrm{n}=77), 80 \%$ $(\mathrm{n}=86)$ et $72 \%(\mathrm{n}=77)$. D'autres contextes comme une consultation de première contraception ou un diagnostic de tuberculose n'engendraient que peu de prescriptions théoriques d'un dépistage VIH : respectivement $25 \%(\mathrm{n}=27)$ et $42 \%$ $(\mathrm{n}=45)$. De plus, $66 \%(\mathrm{n}=71)$ des médecins rapportaient au moins un obstacle à la proposition du dépistage, dont le manque de formation et l'anxiété d'annoncer un résultat positif.

Conclusion Les généralistes installés en Martinique sont sensibles à la problématique locale du VIH. Ils sont d'importants prescripteurs de tests de dépistage : tant au niveau du nombre qu'au niveau de l'initiative de prescription. Il existe cependant des pratiques erronées et des insuffisances de prescription dans certains contextes. D'autres travaux devront en déterminer les causes afin d'améliorer l'offre de dépistage et participer à l'endiguement de l'épidémie de VIH.

Aucun lien d'intérêt

\section{VIH-03}

Dépistage VIH en médecine générale : quelles sont les occasions manquées ?

M. Pillard (1), A. Frésard (1), A. Gagneux-Brunon (1), F. Lucht (1), R. Charles (2), E. Botelho-Nevers (1)

(1) Service de Maladies Infectieuses et Tropicales, Saint-Etienne,

(2) Département de Médecine Générale, Faculté de Médecine, Saint-Etienne.

Introduction La proportion d'infections VIH diagnostiquées tardivement reste élevée en France. Parmi les patients concernés, la quasi-totalité a pourtant eu un recours aux soins primaires dans les 3 ans précédents le diagnostic. L'objectif était d'évaluer le dépistage VIH en médecine générale.

Matériels et méthodes A partir des présentations cliniques des nouveaux dépistés VIH pris en charge récemment dans notre service, un questionnaire comprenant 12 vignettes cliniques courtes correspondant à des infections asymptomatiques chez des groupes à risque $(\mathrm{GR})(\mathrm{n}=4)$, des situations d'infection chronique VIH symptomatique (ICS) $(n=4)$, des situations de primo-infection $(\mathrm{PI}) \mathrm{VIH}(\mathrm{n}=4)$ et 2 vignettes confondantes a été construit. Le questionnaire a été envoyé aux médecins généralistes (MG) par email via 